\title{
The Nucleotide and Predicted Amino Acid Sequence of the Fusion Protein of Recent Isolates of Canine Distemper Virus in Japan
}

\author{
Kiyoko IWATSUKI, Naoko MIYASHITA, Emi YOSHIDA, Yeon-Sil SHIN, Kenjiro OHASHI, Chieko KAI* and \\ Takeshi MIKAMI \\ Department of Veterinary Microbiology, Faculty of Agriculture, The University of Tokyo, Bunkyo-ku, Tokyo 113, Japan \\ (Received 1 September 1997/Accepted 4 November 1997)
}

ABSTRACT. Analysis of the molecular properties of fusion (F) proteins of field isolates of canine distemper virus (CDV) by
immunoprecipitation analysis revealed an identical molecular mass of F protein of 3 field isolates as well as the Onderstepoort laboratory
strain. Sequencing showed that the F gene of a field isolate (the Yanaka strain) shared $90.1 \%$ and $95.7 \%$ identities with the Onderstepoort
strain at nucleotide and amino acid levels, respectively. All of the 13 cysteine residues and 4 potential asparagine-linked glycosylation
sites were completely conserved amongst these strains. These results indicate that the F proteins is much less heterogeneous than that
observed in the hemagglutinin proteins of CDV.-KEY wORDS: CDV, field isolate, fusion protein.
J. Vet. Med. Sci. 60(3): 381-385, 1998

A number of recent outbreaks of canine distemper (CD) have been observed in dogs worldwide [4, 7-9, 10, 15]. The findings of this infection in vaccinated dogs suggest the emergence of new field canine distemper viruses (CDVs) with different antigenic properties from those of the vaccine viruses $[8,9,21]$.

The morbillivirus infection begins with viral attachment to the cell surface followed by fusion between the virus envelope and cell endosomal membrane [18]. Two viral envelope glycoproteins, hemagglutinin $(\mathrm{H})$ and fusion $(\mathrm{F})$ proteins, are responsible for these processes [19, 24]. Thus, mutations introduced in these two CDV genes are one possible reason for the recent outbreaks of CD.

In our previous study, we first analyzed the alterations of $\mathrm{H}$ proteins of recent field isolates from vaccine viruses, and showed that their genetic properties were significantly distinguishable from the vaccine strains [14]. In the present study, we analyzed the molecular properties of the F proteins among CDV strains in an attempt to understand the possible role of this protein in the epidemiology of the current outbreaks of CD.

The three strains of field isolates, the Ueno, Hamamatsu and Yanaka [14], were analyzed. The Onderstepoort strain was used as a standard laboratory strain. These four strains were cultured in B95a cells [17] which were propagated in RPMI-1640 medium supplemented with 5\% fetal calf serum (FCS) and maintained in RPMI-1640 with $2.5 \%$ FCS.

The antigenisities of these four strains were analyzed by two monoclonal antibodies (MAbs) a-8 and b-8 to the F protein of the FXNO strain [11] by indirect immunofluorescence assay. All of the strains were well recognized by them (data not shown).

A radioimmunoprecipitation (RIP) assay using the MAbs a- 8 and b- 8 was performed as described previously [14].

* Correspondence to: Kai, C., Department of Veterinary Microbiology, Faculty of Agriculture, The University of Tokyo, Bunkyo-ku, Tokyo 113, Japan. The seguence of the $\mathrm{F}$ gene of the CDV Yanaka strain has been assigned DDBJ accession number D87949.
The molecular sizes of the F proteins of the three Japanese field isolates were compared with those of the Onderstepoort strain and showed a doublet of the $\mathrm{F}$ protein bands consisting of $41 \mathrm{kDa}$ of $\mathrm{F}_{1}$ and $60 \mathrm{kDa}$ of $\mathrm{F}_{0}$ in the cell lysates infected with each strain (Fig. 1). No significant differences in reactivities with antibodies or in molecular sizes were observed among these $\mathrm{F}$ proteins.

The cDNA of the Yanaka $\mathrm{F}$ gene was amplified by reverse transcriptase-polymerase chain reaction (RT-PCR), cloned into the plasmid (TA cloning kit; Invitrogen), and sequenced by dye-primer cycle sequencing procedures (Applied Biosystems [ABI]) as described elsewhere. The

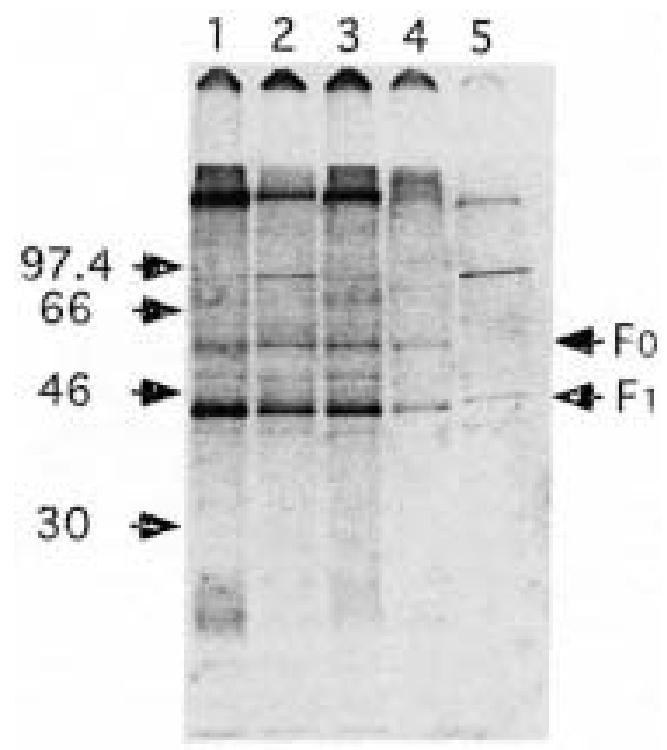

Fig. 1. Immunoprecipitation analysis of CDV protein produced in B95a cells. Lysates of B95a cells infected with field isolates and the Onderstepoort strain of CDV were reacted with anti-CDV MAb, b-8 and analyzed by SDS-PAGE. Lanes: 1, the Onderstepoort strain; 2, the Ueno strain; 3, the Hamamatsu strain; 4, the Yanaka strain; 5, mock-infected B95a cells. 
nucleotide sequence was determined for two clones. There was no difference between the sequences of these clones. To determine the 5'-terminal sequence of the $\mathrm{F}$ gene, the rapid amplification of cDNA end (RACE) method was performed [22]. The following primers were used in these protocols: CDVM5 (5'-TGTCCATCTTTCTATCACAG-3'), F1 (5'-TTAGGGTCCAGGACATAGCA-3'), R3 (5'TCAGAGTGATCTCACATAGG-3') and CDVH22 (5'-TGCTGGACTACCTGAGCCCT-3'); nt 1402 to 1421 of the $\mathrm{M}$ gene, nt -2 to 18 , nt 2074 to 2055 of the F gene and nt 20 to 1 of the $\mathrm{H}$ gene of the Onderstepoort strain of $\mathrm{CDV}$, respectively $[2,3]$. We also prepared the following primers corresponding to the defined Yanaka $\mathrm{F}$ gene and used them for the subsequent determination of the sequence of its undefined regions: CDVF4 (5'-GCCGGGTTTTGGAT CTTTTG-3'), CDVF6 (5'-GGCGGATTTAGTGCTGTGTT5'), CDVH24 (5'-GACTACCTGAGCCCTAAGTT-3'); nt 122 to 103 and nt 169 to 150 of the $\mathrm{F}$ gene and nt 15 to -5 of the $\mathrm{H}$ gene, respectively. CDVM5, F1, CDVH22 and CDVH24 were used for RT-PCR and cloning, and CDVF4 and CDVF6 were used for the RACE method.

When the nucleotide sequence of the $\mathrm{F}$ gene of the Yanaka strain was compared with the database $F$ sequences of the Onderstepoort strain [30] and PDV-2 of CDV (isolated from Baikal seals [28]), no difference in their length of the sequences was observed.

When the nucleotide sequence of the $F$ gene of the Yanaka strain was aligned with those of PDV-2 and the Onderstepoort strains (Fig. 2), three in-frame initiation codons that are possibly used for the open reading frame for the $\mathrm{F}$ protein were observed at positions nt 86 to 88 , nt 266 to 268 , and nt 461 to 463 , the same positions as those of the Onderstepoort strain.

We used the GENETYX-MAC ver. 7.3.1. program (Software Development Co.) for nucleic acid and protein analyses, and the BIORESEARCH/SINCA, ver. 2.0 program (Fujitsu, Japan) for computer-assisted phylogenic analysis by the neighbour-joining method [25] with distance calculation by Kimura's 2-parameter method [16]. The homology between the nucleotide sequences of the F gene of the Yanaka strain and those of the Onderstepoort and PDV-2 strains were estimated to be $90.1 \%$ and $93.6 \%$, respectively. Thus, the genetic relationship was closer between the recent field isolates than between the field isolates and the vaccine virus. The phylogenetic tree also reflected this relationship among the strains (Fig. 3).

The alignment of the predicted amino acid sequence of the F proteins of the three CDV strains is shown in Fig. 4. The signal peptide of the Yanaka strain contains 10 amino acid residues, as does that of the Onderstepoort strain. Thus, the $\mathrm{F}_{0}$ polypeptide of the Yanaka strain would contain 537 amino acids with a calculated molecular weight of $58.6 \mathrm{kDa}$ in the unglycosylated form. The $F_{1} / F_{2}$ cleavage site sequence (RRQRR) and the 13 cysteine residues were completely conserved in all of the three strains. Two hydrophobic regions suspected of being either a fusion or transmembrane domain of the $\mathrm{F}$ protein were conserved among the strains. The amino acid sequence of the Yanaka $\mathrm{F}$ protein shared $95.7 \%$ and $97.4 \%$ identity with the Onderstepoort and PDV-2 strains, respectively.

The analysis of the F protein of a field isolate (Yanaka) in this study showed strong homology of amino acid sequences with another field isolate (PDV-2) and the Onderstepoort strain. The genetic relationship was closer between the recent field isolates, the Yanaka strain and PDV-2, than between the Yanaka and Onderstepoort vaccine strains. These data were in agreement with the result from the $H$ genes [14], and suggest a progressive evolution in wild-type CDV isolates. The significant alteration of PDV$2 \mathrm{~F}$ gene was noted as the lack of the third ATG triplet at positions nt 461 to 463 [28], which is selectively required for in vitro translation of the Onderstepoort $F$ gene [6]. Visser et al. [28] discussed the possible utilization of the second in-frame ATG triplet as initiation codon, or one of the two out-of-frame ATG codons of the PDV-2 F gene in vivo as an in-frame translation initiation codon as a result of RNA "recording". Since the Yanaka strain did not have this mutation, it is not considered to be a common mutation of the $\mathrm{F}$ gene in new field strains.

Four potential asparagine-linked glycosylation sites were conserved among the three strains of the F proteins (Fig. 4) in contrast to the finding that the glycosylation properties of the $\mathrm{H}$ proteins varied between the isolates and the vaccine strains [14]. The similar molecular sizes revealed by the immunoprecipitation analysis may provide the evidence of the same glycosylation properties of these $\mathrm{F}$ proteins. Alkhatib et al. [1] demonstrated that glycosylation might play an important role in the cleavage-dependent activation of the precursor $F_{0}$ protein or in its transport to the subcellular region where the proteolytic cleavage occurs. Our finding of the similar glycosylation properties of the $\mathrm{F}$ proteins suggests that these $F$ proteins have similar characteristics. Thus, the heterogeneity of the $\mathrm{F}$ protein was less than that of the $\mathrm{H}$ protein between field isolates and vaccine strains.

Several reports have demonstrated that the $\mathrm{F}$ protein is sufficient for membrane fusion when recombinant viral glycoproteins are used [1, 12, 23]. Alternatively, it has been shown that the coexpression of the HA and F proteins was necessary for cell fusion [5, 13, 20, 26, 27, 29]. Consequently, several models have been suggested that the HA protein was significant in triggering cell fusion [18, 27]. Although there is some controversy about the role of the $\mathrm{F}$ protein in the transport process, the $\mathrm{F}$ protein apparently plays an important role in cell fusion. The present findings defined that the $\mathrm{F}$ gene is more conserved than the $\mathrm{H}$ gene of field viruses, and thus the alteration of the $\mathrm{H}$ protein might be more likely associated with the recent outbreaks of CD than that of the F protein.

ACKNOWLEDGEMENTS. This study was supported by Grants-in-Aid from the Ministry of Education, Science, Culture and Sports, Japan, by a grant for a pioneering research project in biotechnology from the Ministry of 
Yanaka : AGgGTCCAGGACGCAGCAAGCCAACAGGCCAACCAAGTCCACCAATCCGAGGCCGGGCAGGAACCCCCACGAACAGACAAGCCC MT QCACAACAAAATC

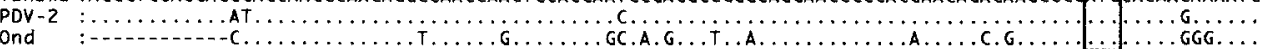
Yanaka: CTCAAAAGATCCAAAACCCGGCCACACACCCGACAAGATCTCCTCCAACAACACAGCACTAAATCCGCCGAGACCAAGACCTCCCAAGGACGATATAGCA

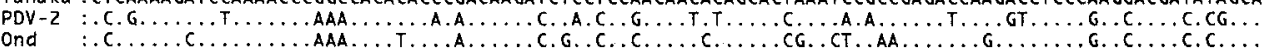

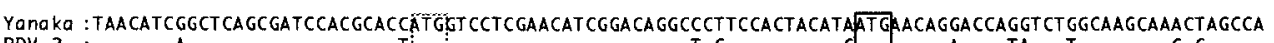

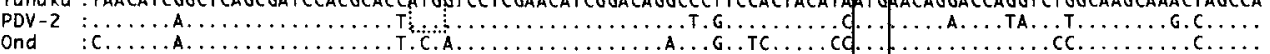
Yanako: CAGATCGgatAACAT CCCGGCTCACAGAGACCACGATGGTATCATCCATCACACACCAGAGAGTTTCAT CCAAGGAGTGAGTTCCCGGTTCAAGAAACGG

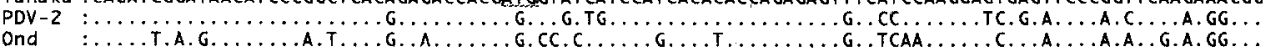

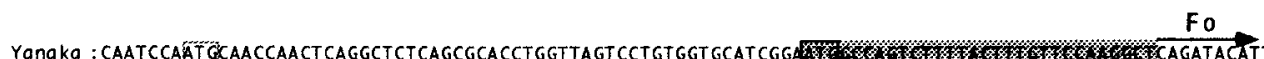

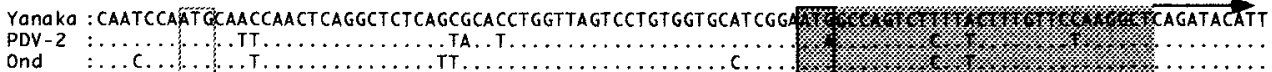
Yanaka: GGAATAATTTGTCAACGATTGGGATTATCGgAACTAACAGTGTCCATTATAAGATCATGACTAGGCCCAGTCACCAGTACTTGGTCATAAAACTAATGCC

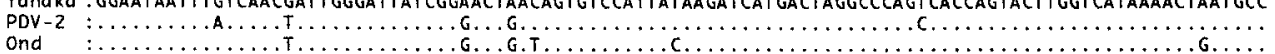
Yanaka: TAATGTTTCACTTATAGATAATTGTACCAAAGCAGAATTAGGTGAGTATGAGAAATTATTAAATTCAGTCCTAGAGCCAATCAACCAAGCTTTGACTCTA

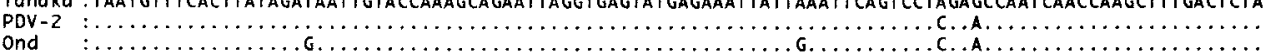

Yanaka : ATGACCAACAATGTGAAGCCCCTACAGTCAGTAGGGTCAGGTAGGAGACAAAGGCGTTITGCAGGAGTGGTGCTTGCAGGT GCAGCTTTAGGGGTGGCCA

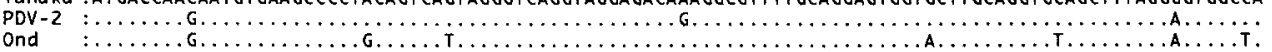
Yanaka : CAGCTGCACAAATCACTGCAGGGATAGCTTTACATCAGTCCAACCT CAATGCTCAAGCAATCCAATCTCTGAGAACTAGCCITGAACAGTCCAACAAGGC

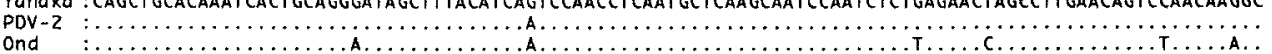

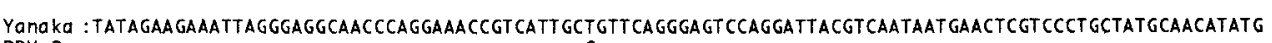

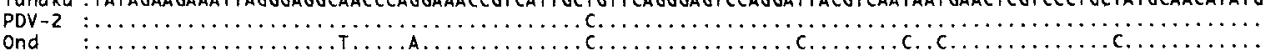
Yanaka :TCGTGTGAATTAGTTGGGCAGAGATTAGgGTTAAAACTGCTTAGGTATTACACCGAGTGGTGTCAATATTTGGCCCGAGTTTACGT GACCCTATTICAG

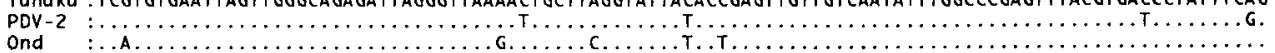
Yanaka: CCGAGATATCAATTCAAGCACTGAGTTATGCTCTTGGGGGAGAAATTCATAAGATACTTGAGAAGITGGGGTATTCTGGAAAT GATATGATTGCAATITT

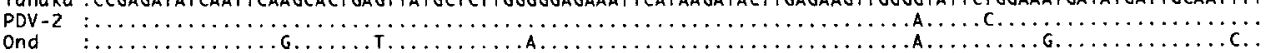
Yanaka : GGAGAGT CGgGgGATAAAAACAAAAATAACTCATGT CGATCT CCCCGGGAAACTCATCATATTAAGTAT CT CATACCCGACITTATCAGAAGTCAAGGGG

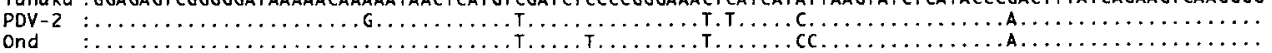

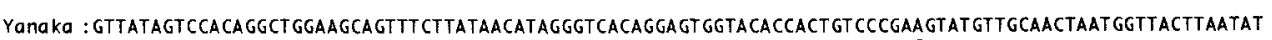

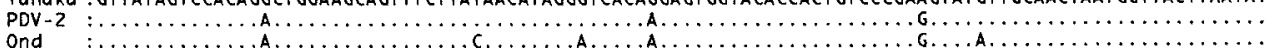
Yanaka : CTAACTTTGATGAGT CATCCTGTGTATTCGTCTCAGAATCAGCCATTT GTAGCCAGAACT CCCTATACCCCATGAGCCCGATTCTACAACAAT GCATTAG

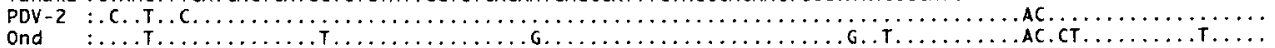
Yanaka : GGGCGACACTTCATCTTGTGCTCGGACCTTGGTGTCTGGGACTATGGGCAACAAGTTTATTCT GTCAAAAGGTAATATCGTTGCAAATTGTGCTTCTATA

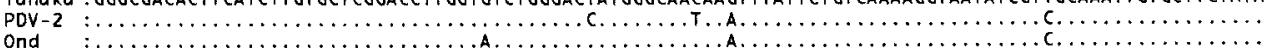
Yanaka: CTATGTAAGTGTTATAGTACAGGCACAATTATTAATCAGAGTCCTGATAAGTTGCTGACATTTATTGCCTCCGATACCTGCCCACTGGITGAAATAGATG

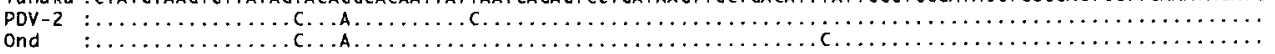
Yanaka : GTGTCACTATTCAAGTTGGAGGGagGCAATACCCTGATATGGTATACGAAAGCAAAGTTGCCTTAGGCCCTGCTATATCACTTGAGAGGTTAGATGTAGG

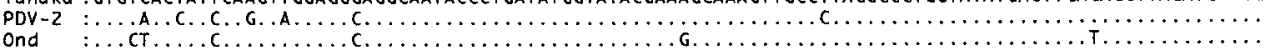

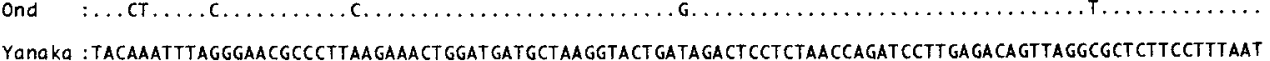

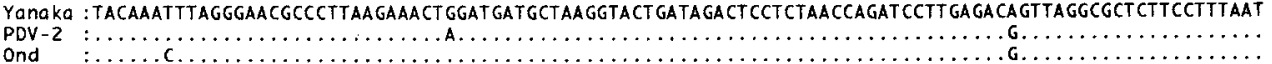

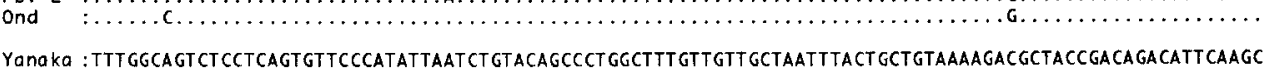

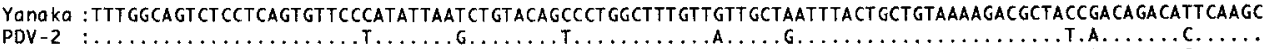

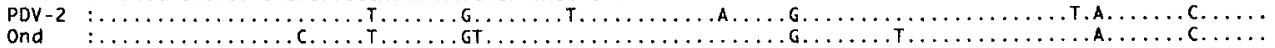

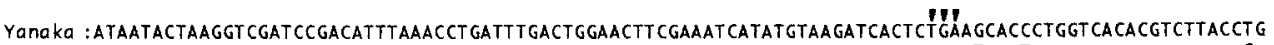

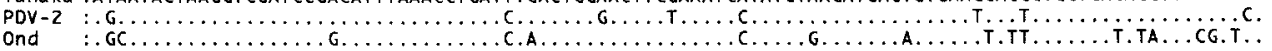

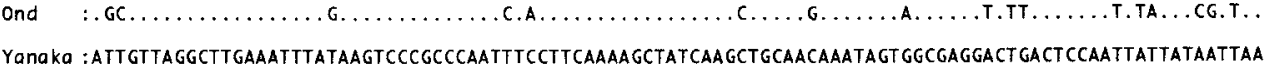

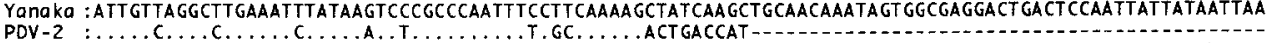

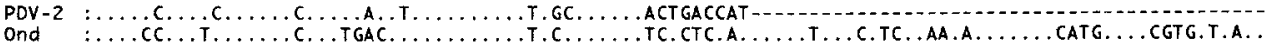
Yanaka :AGAAAACTT 2209 PDV - 2 : :ond

Fig. 2. The entire nucleotide sequence of the F gene of the Yanaka strain (D87949; top line) was compared with those of PDV-2 (D10371; middle line) and the Onderstepoort strain (X65509; bottom line). The shaded area indicates the putative signal sequence. The in-frame translation initiation ATG codons within the sequences upstream of the $\mathrm{F}_{0}$ encoding region are enclosed by rectangles; the out-of-frame ATG codons are enclosed by dotted rectangles. The stop codons are indicated by triangles. Dots represent the nucleotide identity. The numbers of nt positions of the F genes are shown at the right of the lines. 


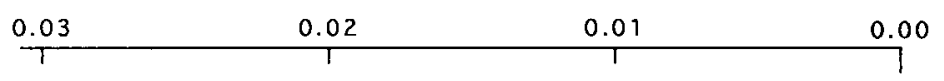

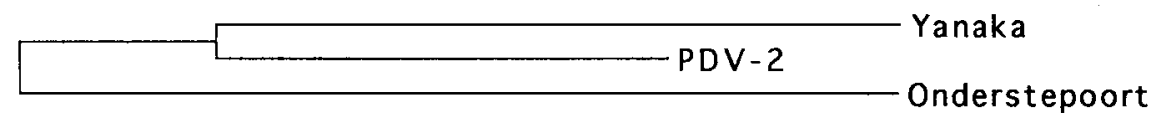

Fig. 3. Phylogenetic relationship of the Onderstepoort strain, PDV-2 strain and Yanaka strain analyzed on their entire protein coding regions of the $\mathrm{F}$ gene by the neighbourjoining method.

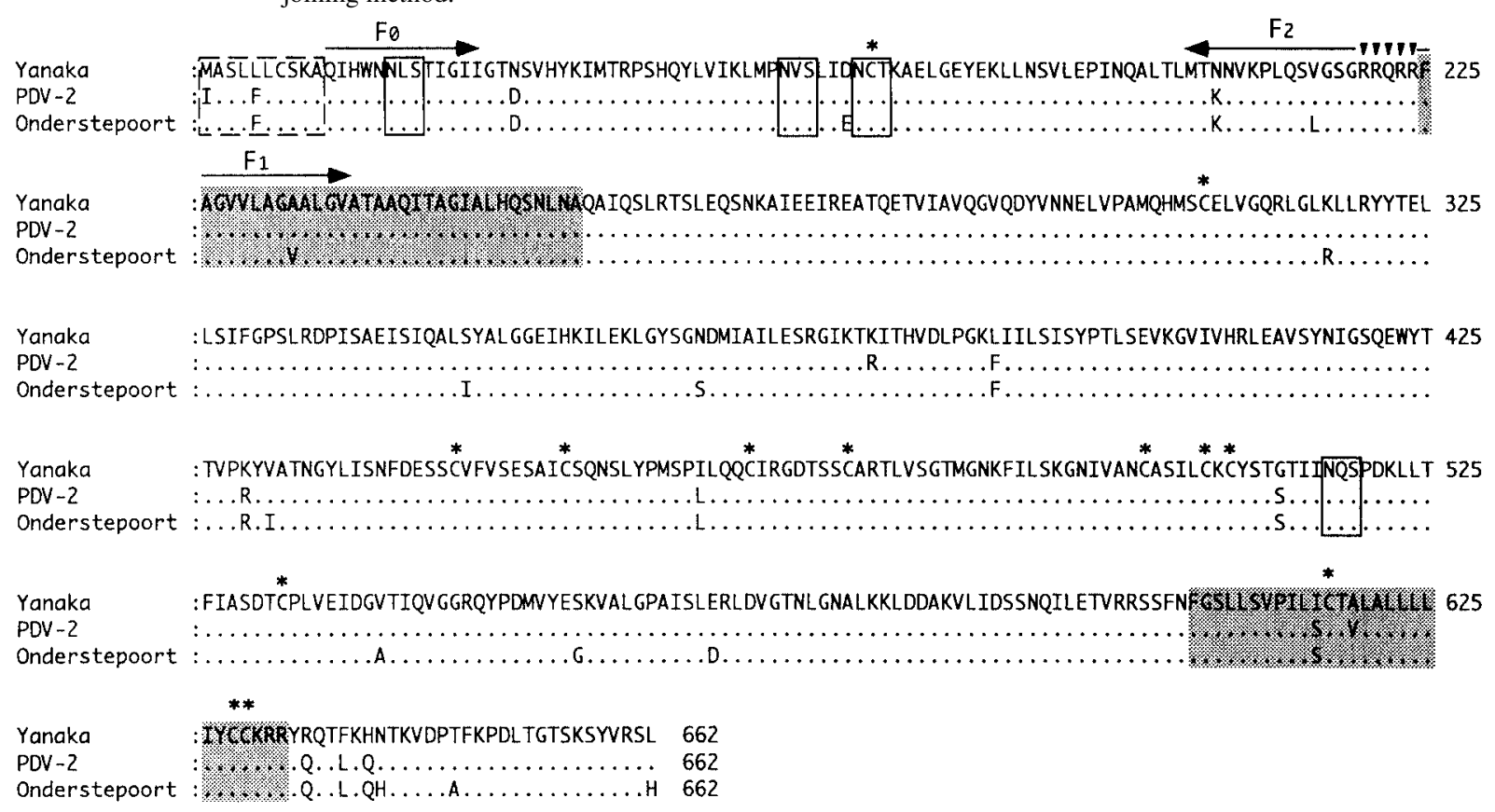

Fig. 4. The predicted amino acid sequence of the F protein of the Yanaka strain (top line) was compared with those of the PDV-2 (middle line) and Onderstepoort strains (bottom line). The signal peptide is enclosed by dotted rectangle. The shaded area indicates the two hydrophobic regions. The potential glycosylation sites are enclosed by rectangles. The putative cleavage site of the protease is indicated by triangles. Asterisks indicate conserved cysteine residues. Dots represent identical amino acids.

Agriculture, Forestry and Fisheries, Japan, and by a grant for Program for Promotion of Basic Research Activities for Innovative Biosciences. K. Iwatsuki is supported by Research Fellowships of Japan Society for the Promotion of Science for Young Scientists.

\section{REFERENCES}

1. Alkhatib, G., Richardson, C. and Shen, S.-H. 1990. Virology 175: 262-270.

2. Bellini, W. J., Englund, G., Richardson, C. D., Rozenblatt, S. and Lazzarini, R. A. 1986. J. Virol. 58: 408-416.

3. Barrett, T., Clarke, D. K., Evans, S. A. and Rima, B. K. 1987. Virus Res. 8: 373-386.

4. Blixenkrone-Møller, M., Svansson, V., Have, P., Örvell, C., Appel, M., Pedersen, I. R., Dietz, H. H. and Henriksen, P. 1993. Vet. Microbiol. 37: 163-173.

5. Ebata, S. N., Côté, M.-J., Yong Kong, C. and Dimock, K. 1991. Virology 183: 437-441.

6. Evans, S. A., Belsham, G. J. and Barrett, T. 1990. Virology
177: $317-323$

7. Gemma, T., Miyashita, N., Shin, Y.-S., Okita, M., Mori, T., Iwatsuki, K., Mikami, T. and Kai, C. 1995. J. Vet. Med. Sci. 57: 761-763.

8. Gemma, T., Watari, T., Akiyama, K., Miyashita, N., Shin, Y.-S., Iwatsuki, K., Kai, C. and Mikami, T. 1996a. J. Vet. Med. Sci. 58: 547-550.

9. Gemma, T., Iwatsuki, K., Shin, Y.-S., Yoshida, E., Kai, C. and Mikami, T. 1996b. J. Vet. Med. Sci. 58: 791-794.

10. Glardon, O. and Stöckli, R. 1985. Schweiz. Arch. Tierheilk. 127: 707-716.

11. Hirayama, N., Senda, M., Nakashima, N., Takagi, M., Sugiyama, M., Yoshikawa, Y. and Yamanouchi, K. 1991. J. Gen. Virol. 72: 2827-2830.

12. Horvath, C. M., Paterson, R. G., Shaughnessy, M. A., Wood, R. and Lamb, R. A. 1992. J. Virol. 66: 4564-4569.

13. $\mathrm{Hu}, \mathrm{X} ., \mathrm{Ray}, \mathrm{R}$. and Compans, R. W. 1992. J. Virol. 66: $1528-1534$

14. Iwatsuki, K., Miyashita, N., Yoshida, E., Gemma, T., Shin, Y.-S., Mori, T., Hirayama, N., Kai, C. and Mikami, T. 1997. J. Gen. Virol. 78: 373-380. 
15. Kai, C., Ochikubo, F., Okita, M., Iinuma, T., Mikami, T., Kobune, F. and Yamanouchi, K. 1993. J. Vet. Med. Sci. 55: 1067-1070.

16. Kimura, M. 1980. J. Molecul. Evol. 16: 111-120.

17. Kobune, F., Sakata, H. and Sugiura, A. 1990. J. Virol. 64: 700-705.

18. Lamb, R. A. 1993. Virology 197: 1-11.

19. Morrison, T. G. 1988. Virus Res. 10: 113-136.

20. Moscona, A. and Peluso, R. W. 1991. J. Virol. 65: 27732777.

21. Okita, M., Yanai, T., Ochikubo, F., Gemma, T., Mori, T., Maseki, T., Yamanouchi, K., Mikami, T. and Kai, C. 1997. J. Comp. Pathol. 116: 403-408.

22. Oshikamo, R., Tohya, Y., Kawaguchi, Y., Tomonaga, K., Maeda, K., Takeda, N., Utagawa, E., Kai, C. and Mikami, T.
1994. J. Vet. Med. Sci. 56: 1093-1099.

23. Paterson, R. G., Hiebert, S. W. and Lamb, R. A. 1985. Proc. Natl. Acad. Sci. U.S.A. 82: 7520-7524.

24. Rima, B. K. 1983. J. Gen. Virol. 64: 1205-1219.

25. Saitou, N. and Nei, M. 1987. Mol. Biol. Evol. 4: 406-425.

26. Sakai, Y. and Shibuta, H. 1989. J. Virol. 63: 3661-3668.

27. Tanabayashi, K., Takeuchi, K., Okazaki, K., Hishiyama, M. and Yamada A. 1992. Virology 187: 801-804.

28. Visser, I. K. G., van der Heijden, R. W. J., van de Bildt, M. W. G., Kenter, M. J. H., Örvell, C. and Osterhaus, A. D. M. E. 1993.J. Gen. Virol. 74: 1989-1994.

29. Wild, T. F., Malvoisin, E. and Buckland, R. 1991. J. Virol. 72: 439-442.

30. Wild, T. F., Bernard, A., Spehner, D., Villeval, D. and Drillien, R. 1993. Vaccine 11: 438-444. 